\title{
WEIGHING THE EVIDENCE FOR THE ABUNDANT-CENTER HYPOTHESIS
}

\author{
Tad A. Dallas ${ }^{1, *}$, Luca Santini ${ }^{2,3}$, Robin Decker $^{4}$, and Alan Hastings ${ }^{5,6}$ \\ ${ }^{1}$ Department of Biological Sciences, Louisiana State University, Baton Rouge, USA \\ ${ }^{2}$ Department of Environmental Science, Institute for Wetland and Water Research, Faculty of \\ Science, Radboud University, PO Box 9010, NL-6500 GL Nijmegen, The Netherlands \\ ${ }^{3}$ National Research Council, Institute of Research on Terrestrial Ecossystems (CNR-IRET), Via \\ Salaria km 29.300, 00015, Monterontondo (Rome), Italy \\ ${ }^{4}$ Department of Integrative Biology, University of Texas, Austin, USA \\ ${ }^{5}$ Department of Environmental Science and Policy, University of California, Davis, USA \\ ${ }^{6}$ Santa Fe Institute, Santa Fe, NM, USA
}

\begin{abstract}
The abundant-center hypothesis posits that species density should be highest in the center of the geographic range or climatic niche of a species, based on the idea that the center of either will be the area with the highest demographic performance (e.g., greater fecundity, survival, or carrying capacity). While intuitive, current support for the hypothesis is quite mixed. Here, we discuss the current state of the abundant-center hypothesis, highlighting the relatively low level of support for the relationship. We then discuss the potential reasons for this lack of empirical support, emphasizing the inherent ecological complexity which may prevent the observation of the abundant-center in natural systems. This includes the role of non-equilibrial population dynamics, species interactions, landscape structure, and dispersal processes, as well as variable data quality and inconsistent methodology. The incorporation of this complexity into studies of the distribution of species densities in geographic or niche space may underlie the limited empirical support for the abundant-center hypothesis. We end by discussing potentially fruitful research avenues. Most notably, we highlight the need for theoretical development and controlled experimental testing of the abundant-center hypothesis.
\end{abstract}

Key words: Abundant-center, Distance-abundance, Macroecology, Niche, Species distribution.

\section{INTRODUCTION}

Based on observational evidence, early ecologists formulated the hypothesis that species abundance (or perhaps more accurately, species density) should be highest in the center of the species geographic range (geographic abundant-center; Brown 1984; Sagarin and Gaines 2002). This makes the assumption that the geographic range is centered on the niche optimum. One way this could occur is through spatially-autocorrelated environmental conditions, the absence of dispersal limitation, and equilibrial population density within each local population across the landscape. This allows for the translation of geographic performance into niche space, where species growth rates are highest where conditions are the most favorable, coinciding with the center of their geographic distributions (Gaston 2009; Sagarin

* Corresponding author: tad.a.dallas@gmail.com and Gaines 2002). However, this may be an oversimplification, as it assumes a coherent environmental gradient, a lack of strong density-dependence, equilibrium population dynamics, no dispersal limitation, and the absence (or unimportance) of competition, predation, mutualism, and parasitism in regulating population demography. It is perhaps due to these strong assumptions that the abundant-center hypothesis has received such mixed empirical support (Pironon et al. 2017; Sagarin et al. 2006; Dallas et al. 2017; Santini et al. 2019). Recently, it was proposed that variation in species density should not be viewed in geographic space, but instead of species' niche space (niche abundant-center; Martínez-Meyer et al. 2013). This helps to remove the confounding effect of spatial autocorrelation on population processes, and links species density more closely with species abiotic tolerances (i.e., the niche). However, 
few studies of the niche abundant-center hypothesis have been performed to date (but see Waldock et al. 2019; Dallas et al. 2017; Martínez-Meyer et al. 2013; Santini et al. 2019). Here, we review evidence for abundant-center ideas, discuss the potential reasons for variability in support, outline existing conceptual issues, and highlight potentially fruitful open questions related to the abundant-center hypothesis.

\section{WHAT IS THE EVIDENCE FOR THE} ABUNDANT-CENTER HYPOTHESIS?

Questions surrounding the spatial distribution of species density are central to population ecology and biogeography, leading to a great interest in understanding the constraints on population size across spatial and environmental gradients (Hengeveld and Haeck 1982; Bell 2000). This interest has generated numerous hypotheses concerning the distribution of abundance across a species geographic range (Hengeveld and Haeck 1982) e.g., the geographic abundant-center hypothesis. Support for the geographic abundant-center hypothesis from large scale analyses can vary from $10 \%$ (Dallas et al. 2017) to values around 50\% (Pironon et al. 2017) of examined species. A systematic meta-analysis is outside of the scope of the current review, and is perhaps redundant considering the two other comprehensive reviews on the topic (Sagarin and Gaines 2002; Pironon et al. 2017). Numerous studies exist examining geographic abundant-center relationships for single or small set of species (Scrosati and Freeman 2019; Samis and Eckert 2007; Feldhamer et al. 2012; Virgós et al. 2011). These studies tend to find more positive support for abundant-center relationships, potentially as a result of increased sampling effort in these more focused studies, or due to a publication bias toward species providing support for the hypothesis.

While the abundant-center hypothesis was first conceptualized with respect to species' geographic distributions, a recent effort has suggested that species should instead be more dense in the center of their environmental niche space (niche abundant-center; Martínez-Meyer et al. 2013). The underlying idea here is to link the species' niche to species performance curves from physiological ecology, in order to link individual survival or fecundity to niche axes. This makes intuitive sense, as individuals located towards the middle of the species environmental tolerances may have higher survival or fecundity rates than individuals towards the niche margins (but see Pironon et al. 2017) for a thorough discussion of this idea). Defining the abundant-center hypothesis in terms of species' niche distances is a useful step, as it removes the conflation of geographic space and climate, and more closely connects with niche theory (Santini et al. 2019). To date, this revised niche abundant-center hypothesis has received approximately the same level of support as the original ( $20 \%$ of studied species; Table 1). For instance, Dallas et al. (2017) and Santini et al. (2019) found limited support for abundant-center relationships given an examined set of 1419 and 108 species respectively, and support differed depending on if the abundant-center is examined in geographic- $15.7 \%$ of species in Santini et al. (2019) and $13.3 \%$ of species in Dallas et al. (2017) —or environmental niche space-17.6\% of species in Santini et al. (2019) and 10.3\% of species in Dallas et al. (2017). Interestingly, some species may also have higher densities on the edge of their geographic distributions $(22.2 \%$ of the species in Santini et al. (2019) and 10\% of species in Dallas et al. (2017) or climatic niches- $12 \%$ of species in Santini et al. (2019) and 8\% of species in Dallas et al. (2017). The prevalence of species following the opposite pattern to the predicted abundant-center is difficult to quantify, as previous systematic reviews have not reported this information (Sagarin and Gaines 2002), or methodological choices prohibit this from even being possible (Waldock et al. 2019). A recent study on North American bird species claims to provide strong support for niche abundant-center relationships (Osorio-Olvera et al. 2020), which varied between $16-45 \%$ depending on the approach (Osorio-Olvera et al. 2020). In Table 1, we report the results where the niche was defined as the MVE of the first three PCA axes, which found support for abundant-center relationships in 200 out of 442 bird species ( $45 \%$ of species), but go into further detail on methodological decisions and abundant-center support elsewhere (Dallas, et al. 2020). This level of variation in support may stem from the taxonomic diversity of species analyzed, methodological choices in estimating abundant-center relationships, or due to publication biases promoting evidence in favor of the hypothesis.

Despite the mixed support and low predictive power of abundant-center relationships in geographic or niche space, there remains an inherent interest in the hypothesis. The sustained interest in the abundant-center hypothesis may stem from the conceptu- 
Table 1: A review of existing support for the abundant-center hypothesis suggests that approximately $20 \%$ of species demonstrate a relationship between species density and distance to a species geographic range or climatic niche center.

\begin{tabular}{|l|c|c|c|c|}
\hline Source & System & Niche & Species $(n)$ & Support $\left(n_{+}\right)$ \\
\hline Sagarin and Gaines (2002) & mixed* & - & 121 & 56 \\
\hline Freeman and Beehler (2018) & birds & - & 17 & 2 \\
\hline Waldock et al. (2019) & reef fish & + & 181 & 56 \\
\hline Dallas et al. (2017) & birds, mammals, fish, \& trees & $+/-$ & 1419 & 118 \\
\hline Martínez-Meyer et al. (2013) & birds \& mammals & $+/-$ & 11 & 10 \\
\hline Rivadeneira et al. (2010) & crabs & - & 5 & 2 \\
\hline Pironon et al. (2015) & plants & $+/-$ & 3 & 0 \\
\hline Santini et al. (2019) & birds and mammals & $+/-$ & 108 & $5-20^{* *}$ \\
\hline Osorio-Olvera et al. (2020) & birds & & 442 & $200^{* * *}$ \\
\hline & & & & \\
\hline Grand total & & & 2307 & 464 \\
\hline
\end{tabular}

*: Sagarin and Gaines (2002) was a meta-analysis combining 22 articles

$+/-$ : study tested for abundant-center in both niche and geographic space

**: Santini et al. (2019) used 9 different distance measures, range of support provided, and the upper value was used in the table totals. ***: Here, we report the relationship using the first three PCA axes and estimating the niche using minimum volume ellipsoids (MVEs). See Osorio-Olvera et al. (2020) for more information, and Dallas, Pironon, and Santini (2020) for a critique.

al simplicity and intuitive nature of the relationship. Further, the implications of the abundant-center relationship provide a means to estimate areas of high conservation priority using information on species occurrence data (Oliveira et al. 2009; Manthey et al. 2015; Boakes et al. 2018). The ability to estimate species density from species occurrence data is a long-standing goal in ecology, as occurrence data are far more plentiful than species abundance data (Ashcroft et al. 2017). However, it remains unclear if models which use species occurrence data to estimate species density can be predictive, as there is mixed evidence for a link between habitat suitability estimated from species distribution models trained on species occurrence data and species densities (Weber et al. 2017; Ashcroft et al. 2017; Dallas et al. 2018; Santini et al. 2019). In addition to examining empirical support for abundant-center relationships, linking the degree of support more closely to species ecology is important. Specifically, understanding the phylogenetic and trait correlates of the abundant-center relationship may provide insight into the drivers of the observed mixed support for the abundant-center hypothesis. Aspects related to species population demography and dispersal processes may influence the extent to which species follow abundant-center relationships, and quantifying these effects may help identify species most (or least) likely to result in abundant-centers. A series of other potential drivers exist, which may contribute to the disconnect between species environmental suitability and species densities. Incorporating these potential drivers will provide a degree of ecological realism and may promote further theoretical development and empirical understanding of abundant-center relationships.

\section{WHAT ARE THE POTENTIAL ECOLOGICAL DRIVERS} OF THE VARIATION IN SUPPORT?

Despite this strong interest in mechanism, studies of the abundant-center hypothesis have traditionally lacked a strong theoretical basis (as discussed in Osorio-Olvera, Soberón, and Falconi 2019; Holt 2019; Dallas and Santini 2020), or empirical support (Table 1). This suggests that - under the assumption that the relationship can exist in controlled systems - variation in support may be driven by unmeasured environmental variation or factors that influence species density apart from niche limits. It is important to note that even in cases where abundant-center relationships are found, these relationships tend to be extremely weak, suggesting that distance from the niche center cannot be used to predict species densities (Dallas and Hastings 2018). There are many reasons for this, many of which relate to how the niche and corresponding center are estimated (discussed further in in the next section). Here, 
we highlight the potential ecological factors which limit the detectability and applicability of the abundant-center relationship to natural populations. These factors, which may drive the low empirical support for abundant-center relationships currently, may be incorporated into future studies, leading to clarifications or refinements on the existing abundant-center hypothesis.

\section{Stochasticity}

Populations are typically not in equilibrium, but fluctuate over time (Lande et al. 2003; Black and McKane 2012) due to stochasticity and flow of individuals between populations through immigration and emigration to and from nearby habitat patches. Within a single population, demographic and environmental stochasticity move populations away from equilibrium (Lande et al. 2003). This is important for at least two reasons. First, demographic stochasticity-variation in species numbers due to probabilistic birth-death processes-influences small populations more strongly, creating inherent spatial variation in species densities over time across the species range. Secondly, environmental stochasticity-in which environmental variation influences probabilistic birthdeath processes - may disproportionately influence some populations in a species range more than others (Ellner et al. 2016). Species' niche axes may only capture static environmental tolerances, failing to incorporate the role of temporal autocorrelation in environmental conditions and the existence of rare climatic events which can have large impacts on species densities (Ellner et al. 2016). This presents a clear area for theoretical development, as existing models used in support of abundant-center theory do not incorporate stochasticity (Osorio-Olvera et al. 2019), and the incorporation of stochasticity strongly influences resulting abundant-center relationships (Dallas and Santini 2020).

\section{Dispersal limitation}

In geographic space, barriers to dispersal can limit where species occur. Reflecting boundaries such as impassable streams serve may influence the spatial distribution of species density by increasing species densities at barriers, and by not allowing access to suitable geographic areas beyond the dispersal barriers (Nislow et al. 2011). This can further influence the estimation of a species' niche, as the niche is commonly defined using data on geographic occurrence points of a species. Species dispersal can be informed by social cues, habitat patch quality, and climatic events (Clobert et al. 2009; Reed et al. 1999; Jacob et al. 2015; Travis and Dytham 1999), suggesting that population density will non-randomly fluctuate, independent of any abundant-center constraints. Further, dispersal processes may create spatially-aggregated clusters of individuals which may not truly represent the potential distribution of the species, but are a result of limited dispersal distance (Condit et al. 2000). The spatial arrangement of habitat patches, dispersal distance and barriers, and the distribution of populations in geographic or environmental space is also important, as frequent dispersal events may result in spatial synchrony (Paradis et al. 1999), potentially influencing support for abundant-center relationships. Finally, species with limited dispersal ability may occupy only a small part of their fundamental niche space even in the absence of dispersal barriers. This may still lead to abundant-center relationships, but only if the species has a strong core number of populations aggregated in geographic or environmental space. That is, if a species with poor dispersal ability has disjoint populations, this could create a bimodality in the resulting abundant-center relationship, where species are most abundant in the small regions of geographic or environmental space where populations are established.

\section{Species interactions}

Species do not exist in isolation from one another, but interact through competition, predation, mutualism, and parasitism. These interactions are geographically variable (Early and Keith 2019), such that even directly including the density of an interacting species in an abundant-center analysis may not capture the reality of the system. Presently, no abundant-center hypothesis study has explicitly accounted for the influence of these antagonistic and mutualistic forces. Despite this, ecologists have long recognized that competition (Greiner La Peyre et al. 2001; Lawlor 1979), predator-prey dynamics (Blasius et al. 1999; Stenseth et al. 1997), mutualistic interactions (Holland et al. 2002), and parasitism (Hochberg and Holt 1990; Hudson et al. 1998) can all strongly influence species population dynamics. Related to the importance of species interactions to determining local population size, the order of species' arrival to a site can determine competitive outcomes and strongly influence species density (Strauss 1991; 
Fukami 2015). However, these priority effects (or historical contingencies) are difficult to incorporate into abundant-center analyses. Most often, this is due to the limited data available on the order of species arrival, though the conceptual framework used to test the abundant-center hypothesis also doesn't provide a clear way to incorporate these dynamic processes into the expected relationship between species density and geographic or niche distance.

\section{Species traits}

Even if we assume species do exist in relative isolation, responding to environmental variation independently from other species, species traits may influence abundant-center relationships. Understanding how species-level abundant-center relationships are influenced by phylogenetic relationships and traits is an important research frontier (Dallas et al. 2017; Santini et al. 2019). For example, examining North American birds, Osorio-Olvera et al. (2020) found evidence that body mass, migratory status, and habitat (aquatic or terrestrial) influenced abundant-center relationships, while Dallas et al. (2017) failed to detect an effect of body size on abundant-center relationships in birds, trees (measured as height), fishes, or mammals. Species traits are expected to influence abundant-center relationships through the lens of demographic performance and distributional effects. That is, traits like body mass or species leaf area may be strongly related to maximum demographic rates (Duncan et al. 2007), while traits relating to species dispersal distance (e.g., seed mass) may collectively influence the range and distributions of observable species densities (Soudzilovskaia et al. 2013; Harpole and Tilman 2006).

\section{Anthropogenic effects}

Human effects on landscapes may strongly influence species abundances through the effects of disturbance, exploitation, and land use changes (Sykes et al. 2019; Benítez-López et al. 2010; Benítez-López et al. 2017). Changing land use can strongly influence species densities by altering resource availability or shifting community composition, as species will vary in their ability to tolerate human disturbance. However, the response of species densities to human-induced environmental changes can be mixed, as humans can often provide food supplementation, can act as predator deterrent, leading to increased density (Sorace 2002; Jesse et al. 2018; Wang et al. 2015;
Rouco et al. 2019; Šálek et al. 2015), while direct disturbance, exploitation, shifts in competitive community structure, mating opportunities, and reduced access to resources can also reduce population density (Lemoine et al. 2007; Benítez-López et al. 2010; Benítez-López et al. 2017). Anthropogenic barriers can alter the distribution and abundance of migratory species (Said et al. 2016). Humans have also altered the size and shape of species geographic ranges (Di Marco and Santini 2015; Channell and Lomolino 2000), an effect which would obviously influence the detection of the geographic or niche center and thus abundant center-relationships. Further, intensity of human effects on a landscape are not likely to be uniformly distributed across a species geographic range or within climatic niche space (Sanderson et al. 2002; Benítez-López et al. 2019), leading to considerable variation and potential divergence from abundant-center relationships. Incorporating human population density or land use change more explicitly into abundant-center ideas could help disentangle how disturbance can influence the spatial distribution of species densities. However, whether or not these variables represent true niche axes depends on how the niche is conceptually defined, as niche concepts differ in the incorporation of abiotic and biotic axes.

\section{POTENTIAL METHODOLOGICAL DRIVERS} OF VARIATION IN SUPPORT

Apart from the above mechanisms, there are also a number of existing issues with how support for the abundant-center hypothesis is quantified. For instance, measuring species densities instead of demographic rates assumes that the population density is a proportional representation of demographic processes, which is not necessary in non-equilibrial populations where demographic and environmental stochasticity play a strong role. Further, the original formulation of the abundant-center hypothesis did not explicitly operationalize the estimation of "center". That is, some researchers relate species density to $a$ ) the distance from each sampled population to the geographic (or niche) center, $b$ ) distance to the geographic (or niche) edge, or $c$ ) one of numerous other measures (e.g., Santini et al. (2019) use nine different measures). Many of these measures require estimation of the center of a species geographic (or niche), as well as the use of an appropriate distance measure to quantify distance of a population from geographic range (or niche) center (Dallas et 
al. 2017; Soberón et al. 2018). Aggregated or incomplete sampling of a species geographic range or climatic niche area then represents a clear issue in the detection of abundant-center relationships (Pironon et al. 2017), as this may bias the estimation of the geographic range (or climatic niche) center.

\section{Distance measures and niche delineation}

With respect to distance measures, numerous methods have been used, with Mahalanobis distance being a current favorite since it incorporates the covariance between spatial or climatic axes (Soberón et al. 2018; Osorio-Olvera et al. 2019). However, it is important to note that some distance measures may be highly correlated (Dallas et al. 2018) —while others are not (Santini et al. 2019) - suggesting that criticisms based on distance measure used have the potential to be meaningful or not. This creates a clear issue, as different measures may produce drastically different results, and researchers wishing to support abundant-center ideas could simply select a combination of measures which provide the strongest degree of support. Further, the methodological decision of how to delineate geographic range (or niche) boundaries is another consideration, as each method of delineating a species geographic range or climatic niche makes assumptions. There are at least two conceptual approaches to this.

The first involves training a species distribution model on environmental covariates, and using this model to delineate the species geographic range or climatic niche space. This approach may get around spatial sampling biases and more accurately capture the species' niche and geographic distribution, but suffers from existing issues in species distribution modeling, such as the identification of relevant niche axes used as covariates, the choice of modeling approach (e.g., presence-background versus presence only approaches, different modelling algorithms (Norberg et al. 2019), and thresholding the continuous prediction of species distribution models to binary estimate for range delineation. Additionally, niche models attempt to model the Grinellian niche (e.g. abiotic component), while the Eltonian niche (e.g. biotic component) is generally only partly considered or entirely disregarded (Soberón and Nakamura 2009). Finally, these models rely on the assumption that species are in equilibrium with the environment, but this assumption is often violated biasing niche estimates (Faurby and Araújo 2018).
A second approach simply uses the sampled species occurrence points to delineate the geographic range and corresponding environmental niche space. Methods of delineating species range from occurrence data such as convex hulls may overestimate range size and bias range center estimation (Soberón et al. 2018), but benefit from being simply defined and not requiring parameterization. For instance, alpha hulls offer another way to delineate range, but they are highly sensitive to parameterization (Joppa et al. 2016). Lastly, estimation methods tend to assume a particular shape of the niche (e.g., ellipsoidal), while identifying the true shape of the niche would require far more data and far fewer assumptions.

\section{Defining the niche}

One of the greatest strengths of the abundant-center hypothesis is the relation of species performance curves along environmental gradients to the concept of the species' niche and corresponding geographic projection. However, this strength is complicated by the multiple definitions of the species' niche (Leibold 1995; Colwell and Rangel 2009). Some of these niche definitions are at odds with abundant-center ideas. For instance, Hutchinson (1957) described the niche as a persistence boundary, not a continuous surface of suitability or demographic performance. Even when the niche may be estimated perfectly in -dimensional space, a variety of niche shapes could be estimated, with some resulting in a center that is not actually contained within the niche, or if the niche is discontinuous. This is true for the realized niche, which is the form of the niche that data on the known geographic distributions of species allows us to estimate, and may not be the case for the fundamental niche. For instance, there may be regions of climatic niche space that are not part of the niche, but are contained within niche (Blonder 2016). Finally, the identification of appropriate niche axes can strongly influence resulting support for abundant-center relationships, as Osorio-Olvera et al. (2020) demonstrated by defining the niche using all possible combinations of 2-3 climatic niche axes for a set of species.

Assuming species density, carrying capacity, or growth rates do peak in the center of a species' niche, there remains the issue of which climatic covariates best define the niche. Most studies of niche abundant-center relationships use temperature and precipitation variables to define the niche space, which may only capture mean conditions, and does not include 
information on habitat quality, predator density, or other factors which could strongly influence population density and temporal dynamics. These climatic covariates are able to capture species distributions based on occurrence data well (Norberg et al. 2019), but may not be able to accurately estimate species densities (Dallas and Hastings 2018).

\section{Data considerations}

Data quality is a common issue affecting the empirical examination of numerous macroecological laws, and the abundant-center hypothesis is no exception. Especially in macroecology, data are often difficult to obtain, as spatial and temporal coverage vary considerably, different data sources may not be directly comparable, and the inherent presence of sampling and detection biases (Knouft 2018). Studies aimed at examining consistency of abundant-center support to methodological decisions are important (Santini et al. 2019), as inconsistent methodology can also strongly influence the resulting level of support (or non-support).

A critical first step in addressing the influence of methodological decisions is to release all code and data to reproduce the analyses, allowing others to modify the original approach and determine change in support (Dallas et al. 2017; Dallas and Hastings 2018; Waldock et al. 2019; Osorio-Olvera et al. 2020). However, we should also bear in mind that finding a stronger abundant-center relationship does not validate a methodological approach or the quality of a data source, as the degree of support (or non-support) for the abundant-center relationship is not inherently a reflection on data quality or the suitability of a given method.

What counts as abundant-center evidence?

Perhaps a more important current limitation is what we currently consider as evidence supporting the abundant-center idea. Some studies have used rank correlation coefficients between distance and density to address the significance and strength of the abundant-center relationship (Santini et al. 2019), while others have used a classification approach based on threshold values (Waldock et al. 2019). That is, if species density at the range margins is less than some percentage the density of a species in the geographic or niche center, a species is said to follow an abundant-center distribution. This makes a rigorous meta-analysis of support for the abundant-center hypothesis difficult, as thresholds for statistical support differ greatly, and authors quantify "support" in many different ways.

\section{WHAT SHOULD WE DO NEXT?}

In light of these existing issues and the need for further theoretical development, it is worth outlining important next steps in the study of the abundant-center hypothesis. This list is neither exhaustive nor prescriptive, as this area has been, and will likely continue to be, of great interest to ecologists and biogeographers with different research approaches. The continued development of theory (Holt 2019; Dallas and Santini 2020), as well as the use of controlled experimental approaches (e.g., microcosms), represent two useful advances to the study of abundant-center ideas. The combination of observational, experimental, and theoretical approaches will aid in addressing the numerous questions which currently exist, including:

1. How do demographic and environmental stochasticity influence the distribution of species density in geographic or niche space?

2. Is there a species trait basis for variation in support for the abundant-center hypothesis?

3. What is the influence of human impacts on the support for the abundant-center hypothesis?

4. What are the relative roles of niche requirements and species interactions (e.g., predation, competition, parasitism) on abundant-center relationships?

5. Do mutualistic species tend to both follow abundant-centers with respect to their own niche requirements, or as a function of interactor density (e.g., are plant-pollinator interactions driven by climate or availability of partners)?

6. Instead of simple measures of population density, how do demographic rates vary as a function of distance from the geographic range or climatic niche center? (see Pironon et al. 2017 for examples of this approach)

7. What is the role of seasonal environmental fluctuations to abundant-center 
ideas? Seasonally fluctuating environments will move populations in niche space, resulting in different estimates of distance and variability in the relationship between distance and density.

8. How do abundant-center relationships change temporally? Species with strong seasonal dynamics may occupy a different range or attain variable densities as a function of phenology and environmental drivers which may lead to a seasonally variable relationship between species density and distance from the geographic range or climatic niche (Dallas and Hastings 2018).

9. How does global change alter our ability to detect an abundant-center pattern? Changes to environmental conditions shift the niche center in space, yet demographic responses may be lagged behind.

But what if the goal of the abundant-center hypothesis is not to predict species densities, but simply to document the decline of species density within a species geographic range or climatic niche (i.e., to document a pattern)? Many similar macroecological and biogeographical laws exist, where the current goal is to gauge support for the relationship across different taxa and environments (Pironon et al. 2017). This is certainly a worthwhile endeavor, provided the conclusions are tempered by considering the degree of statistical support for abundant-center relationships, which are typically quite low Pironon et al. (2017).

\section{ConCluding THOUGHTS}

It is important to not become dogmatic in the assessment of abundant-center ideas. While intuitive, the mixed support for the hypothesis suggests that other factors are important in structuring the spatial distribution of species densities. Even in studies showing support for the abundant-center relationship, the amount of variance in species density explained by the selected distance measure is quite small, which limits the utility of the relationship for prediction, and suggests that other processes may contribute far more strongly to predicting species densities than distance measures. Further conceptual and theoretical development is necessary to understand when we would expect to find abundant-center relationships, and what other factors contribute to controlling species density at a given site. To this end, applying spatial population dynamic models is a fruitful path forward to explore the conditions which promote abundant-center relationships in controlled simulations. Finally, the use of laboratory systems to test abundant-center ideas is a clear research need, as they allow the ability to define the species' niche independent of geographic space and to control the amount of variation present in the system. This not only provides a baseline for how strong abundant-center relationships can be when all other environmental variation is ignored, but would also allow for demonstrations of the relative effects of temporal variation in environmental conditions, species interactions, and dispersal dynamics in structuring species densities across geographic or niche gradients.

\section{ACKNOWLEDGMENTS}

The research was funded by the Academy of Finland and the Jane and Aatos Erkko Foundation. We thank Town Peterson and an anonymous reviewer for their constructive comments on earlier drafts. This work has been performed with funding to T. Dallas from the National Science Foundation (NSF-DEB-2017826).

\section{AUTHOR CONTRIBUTIONS}

All authors contributed to manuscript writing.

\section{DATA ACCESSIBILITY}

There is no data or code associated with this manuscript.

\section{CONFLICT OF INTEREST}

The authors have no conflicts of interest to declare.

\section{REFERENCES}

Ashcroft, M.B., D.H. King, B. Raymond, J.D. Turnbull, J. Wasley, and S.A. Robinson. 2017. Moving beyond presence and absence when examining changes in species distributions. Global Change Biology 23:2929-2940.

Bell, G. 2000. The distribution of abundance in neutral communities. American Naturalist 155:606-617.

Benítez-López, A., R. Alkemade, A.M. Schipper, D.J. Ingram, P.A. Verweij, J.A.J. Eikelboom, and M.A.J. Huijbregts. 2017. The impact of hunting on tropical mammal and bird populations. Science 356:180-183.

Benítez-López, A., R. Alkemade, and P.A. Verweij. 2010. The impacts of roads and other infrastructure on 
mammal and bird populations: A meta-analysis. Biological Conservation 143:1307-1316.

Benítez-López, A., L. Santini, A.M. Schipper, M. Busana, and M.A.J. Huijbregts. 2019. Intact but empty forests? Patterns of hunting-induced mammal defaunation in the Tropics. PLoS Biology 17:e3000247.

Black, A.J., and A.J. McKane. 2012. Stochastic formulation of ecological models and their applications. Trends in Ecology \& Evolution 27:337-345.

Blasius, B., A. Huppert, and L. Stone. 1999. Complex dynamics and phase synchronization in spatially extended ecological systems. Nature 399: 354.

Blonder, B. 2016. Do hypervolumes have holes? American Naturalist 187: E93-E105.

Boakes, E.H., N.J.B. Isaac, R.A. Fuller, G.M. Mace, and P.J.K. McGowan. 2018. Examining the relationship between local extinction risk and position in range. Conservation Biology 32:229-239.

Brown, J.H. 1984. On the relationship between abundance and distribution of species. American Naturalist 124:255-279.

Channell, R., and M.V. Lomolino. 2000. Trajectories to extinction: Spatial dynamics of the contraction of geographical ranges. Journal of Biogeography 27:169-179.

Clobert, J., J.-F. Le Galliard, J. Cote, S. Meylan, and M. Massot. 2009. Informed dispersal, heterogeneity in animal dispersal syndromes and the dynamics of spatially structured populations. Ecology Letters 12:197209.

Colwell, R.K., and T.F. Rangel. 2009. Hutchinson's Duality: The once and future niche. Proceedings of the $\mathrm{Na}$ tional Academy of Sciences USA 106:19651-19658.

Condit, R., P.S. Ashton, P. Baker, S. Bunyavejchewin, S. Gunatilleke, N. Gunatilleke, S.P. Hubbell, R.B. Foster, A. Itoh, J.V. LaFrankie, and H.S. Lee. 2000. Spatial patterns in the distribution of tropical tree species. Science 288:1414-1418.

Dallas, T.A., and A. Hastings. 2018. Habitat suitability estimated by niche models is largely unrelated to species abundance. Global Ecology and Biogeography 27:1448-1456.

Dallas, T.A., R.R Decker, and A. Hastings. 2017. Species are not most abundant in the center of their geographic range or climatic niche. Ecology Letters 20:15261533.

Dallas, T.A., R.R Decker, and A. Hastings. 2018. Multiple data sources and freely available code is critical when investigating species distributions and diver- sity: A response to Knouft (2018). Ecology Letters 21:1423-1424.

Dallas, T.A., S. Pironon, and L. Santini. 2020. Weak support for the abundant niche-center hypothesis in North American birds. BioRxiv doi: https://doi. org/10.1101/2020.02.27.968586.

Dallas, T.A., and L. Santini. 2020. The influence of stochasticity, landscape structure, and species traits on abundant-center relationships. Ecography 43:13411351.

Di Marco, M., and L. Santini. 2015. Human pressures predict species' geographic range size better than biological traits. Global Change Biology 21:2169-2178.

Duncan, R.P., D.M. Forsyth, and J. Hone. 2007. Testing the metabolic theory of ecology: Allometric scaling exponents in mammals. Ecology 88:324-333.

Early, R., and S.A. Keith. 2019. Geographically variable biotic interactions and implications for species ranges. Global Ecology and Biogeography 28:42-53.

Ellner, S.P, D.Z. Childs, and M. Rees. 2016. Environmental stochasticity. Pp. 187-227 in Data-Driven Modelling of Structured Populations. Springer.

Faurby, S., and M.B. Araújo. 2018. Anthropogenic range contractions bias species climate change forecasts. Nature Climate Change 8:252.

Feldhamer, G.A, D.B. Lesmeister, J.C. Devine, and D.I. Stetson. 2012. Golden Mice (Ochrotomys nuttalli) co-occurrence with Peromyscus and the abundant-center hypothesis. Journal of Mammalogy 93:1042-1050.

Freeman, B.G., and B.M. Beehler. 2018. Limited support for the 'abundant center' hypothesis in birds along a tropical elevational gradient: Implications for the fate of lowland tropical species in a warmer future. Journal of Biogeography 45:1884-1895.

Fukami, T. 2015. Historical contingency in community assembly: Integrating niches, species pools, and priority effects. Annual Review of Ecology, Evolution, and Systematics 46:1-23.

Gaston, Kevin J. 2009. Geographic range limits: Achieving synthesis. Proceedings of the Royal Society $B$ 276:1395-1406.

La Peyre, M.K.G., J.B. Grace, E. Hahn, and I.A. Mendelssohn. 2001. The importance of competition in regulating plant species abundance along a salinity gradient. Ecology 82: 62-69.

Hengeveld, R., and J. Haeck. 1982. The distribution of abundance. I. Measurements. Journal of Biogeography 9:303-316. 
Hochberg, M.E., and R.D. Holt. 1990. The coexistence of competing parasites. I. The role of cross-species infection. American Naturalist 136:517-541.

Holland, J.N., D.L. DeAngelis, and J.L. Bronstein. 2002. Population dynamics and mutualism: Functional responses of benefits and costs. American Naturalist 159:231-244.

Holt, Robert D. 2019. Reflections on niches and numbers. Ecography 43:387-390.

Hudson, Peter J, Andy P Dobson, and Dave Newborn. 1998. Prevention of population cycles by parasite removal. Science 282:2256-2258.

Hutchinson, G Evelyn. 1957. Concluding remarks. Cold Spring Harbor Symposium on Quantitative Biology 22:415-427.

Jacob, S., A.S. Chaine, N. Schtickzelle, M. Huet, and J. Clobert. 2015. Social information from immigrants: Multiple immigrant-based sources of information for dispersal decisions in a ciliate. Journal of Animal Ecology 84:1373-1383.

Jesse, W.A.M., J.E. Behm, M.R. Helmus, and J. Ellers. 2018. Human land use promotes the abundance and diversity of exotic species on Caribbean islands. Global Change Biology 24:4784-4796.

Joppa, L.N., S.H.M. Butchart, M. Hoffmann, S.P. Bachman, H.R. Akçakaya, J.F. Moat, M. Böhm, R.A. Holland, A. Newton, B. Polidoro, and A. Hughes. 2016. Impact of alternative metrics on estimates of extent of occurrence for extinction risk assessment. Conservation Biology 30: 362-370.

Knouft, J.H. 2018. Appropriate application of information from biodiversity databases is critical when investigating species distributions and diversity: A comment on Dallas et al. Ecology Letters 21:1119-1120.

Lande, R., S. Engen, and B.-E. Saether. 2003. Stochastic Population Dynamics in Ecology and Conservation. Oxford University Press.

Lawlor, L.R. 1979. Direct and indirect effects of $N$-species competition. Oecologia 43: 355-364.

Leibold, Matthew A. 1995. The niche concept revisited: Mechanistic models and community context. Ecology 76:1371-1382.

Lemoine, N., H.-G. Bauer, M. Peintinger, and K. Böhning-Gaese. 2007. Effects of climate and land-use change on species abundance in a Central European bird community. Conservation Biology 21:495-503.

Manthey, J.D., L.P. Campbell, E.E. Saupe, J. Soberón, C.M. Hensz, C.E. Myers, H.L. Owens, K. Ingenloff, A.T. Peterson, N. Barve, and A. Lira-Noriega. 2015. A test of niche centrality as a determinant of population trends and conservation status in threatened and endangered North American birds. Endangered Species Research 26 (3): 201-8.

Martínez-Meyer, E., D. Díaz-Porras, A.T. Peterson, and C. Yáñez-Arenas. 2013. Ecological niche structure and rangewide abundance patterns of species. Biology Letters 9:20120637.

Nislow, K.H., M. Hudy, B.H. Letcher, and E.P Smith. 2011. Variation in local abundance and species richness of stream fishes in relation to dispersal barriers: Implications for management and conservation. Freshwater Biology 56:2135-2144.

Norberg, A., N. Abrego, F.G. Blanchet, F.R. Adler, B.J. Anderson, J. Anttila, M.B Araújo, T.A. Dallas, D. Dunson, J. Elith, and S.D. Foster. 2019. A comprehensive evaluation of predictive performance of 33 species distribution models at species and community levels. Ecological Monographs 89:e01370.

Oliveira, G. de, J.A.F. Diniz-Filho, L.M. Bini, and T.F.L.V.B. Rangel. 2009. Conservation biogeography of mammals in the cerrado biome under the unified theory of macroecology. Acta Oecologica 35:630638.

Osorio-Olvera, L., J. Soberón, and M. Falconi. 2019. On population abundance and niche structure. Ecography 42:1415-1425.

Osorio-Olvera, L., C. Yañez-Arenas, E. Martínez-Meyer, and A.T. Peterson. 2020. Relationships between population densities and niche-centroid distances in North American birds. Ecology Letters 23:555-564.

Paradis, E., S.R. Baillie, W.J. Sutherland, and R.D. Gregory. 1999. Dispersal and spatial scale affect synchrony in spatial population dynamics. Ecology Letters 2:114-120.

Pironon, S., G. Papuga, J. Villellas, A.L. Angert, M.B. García, and J.D. Thompson. 2017. Geographic variation in genetic and demographic performance: New Insights from an old biogeographical paradigm. Biological Reviews 92:1877-1909.

Pironon, S., J. Villellas, W.F. Morris, D.F. Doak, and M.B. García. 2015. Do geographic, climatic or historical ranges differentiate the performance of central versus peripheral populations? Global Ecology and Biogeography 24:611-620.

Reed, J.M., T. Boulinier, E. Danchin, and L.W. Oring. 1999. Informed dispersal. Pp. 189-259 in Current Ornithology. Springer.

Rivadeneira, M.M., P. Hernáez, J.A. Baeza, S. Boltana, M. Cifuentes, C. Correa, A. Cuevas, E. del Valle, I. Hinojosa, N. Ulrich, and N. Valdivia. 2010. Testing 
the abundant-center hypothesis using intertidal porcelain crabs along the chilean coast: Linking abundance and life-history variation. Journal of Biogeography 37:486-498.

Rouco, C., I.C. Barrio, F. Cirilli, F.S. Tortosa, and R. Villafuerte. 2019. Supplementary food reduces home ranges of european wild rabbits in an intensive agricultural landscape. Mammalian Biology 95:35-40.

Sagarin, R.D., and S.D Gaines. 2002. The 'abundant center' distribution: To what extent is it a biogeographical rule? Ecology Letters 5 (1): 137-147.

Sagarin, R.D., S.D. Gaines, and B. Gaylord. 2006. Moving beyond assumptions to understand abundance distributions across the ranges of species. Trends in Ecology \& Evolution 21 (9): 524-530.

Said, M.Y., J.O. Ogutu, S.C. Kifugo, O. Makui, R.S. Reid, and J. de Leeuw. 2016. Effects of extreme land fragmentation on wildlife and livestock population abundance and distribution. Journal for Nature Conservation 34:151-164.

Šálek, M., L. Drahníková, and E. Tkadlec. 2015. Changes in home range sizes and population densities of carnivore species along the natural to urban habitat gradient. Mammal Review 45:1-14.

Samis, K.E., and C.G. Eckert. 2007. Testing the abundant center model using range-wide demographic surveys of two coastal dune plants. Ecology 88:1747-1758.

Sanderson, E.W., M. Jaiteh, M.A. Levy, K.H. Redford, A.V. Wannebo, and G. Woolmer. 2002. The human footprint and the last of the wild: The human footprint is a global map of human influence on the land surface, which suggests that human beings are stewards of nature, whether we like it or not. BioScience 52:891-904.

Santini, L., S. Pironon, L. Maiorano, and W. Thuiller. 2019. Addressing common pitfalls does not provide more support to geographical and ecological abundant-center hypotheses. Ecography 42:696-705.

Scrosati, R.A., and M.J. Freeman. 2019. Density of intertidal barnacles along their full elevational range of distribution conforms to the abundant-center hypothesis. PeerJ 7:e6719.

Soberón, J., and M. Nakamura. 2009. Niches and distributional areas: Concepts, methods, and assumptions. Proceedings of the National Academy of Sciences USA 106:19644-19650.

Soberón, J., A.T. Peterson, and L. Osorio-Olvera. 2018. A comment on 'species are not most abundant in the center of their geographic range or climatic niche.' Rethinking Ecology 3:13.
Sorace, A. 2002. High density of bird and pest species in urban habitats and the role of predator abundance. Ornis Fennica 79:60-71.

Soudzilovskaia, N.A., T.G. Elumeeva, V.G. Onipchenko, I.I. Shidakov, F.S. Salpagarova, A.B. Khubiev, D.K. Tekeev, and J.H.C. Cornelissen. 2013. Functional traits predict relationship between plant abundance dynamic and long-term climate warming. Proceedings of the National Academy of Sciences USA 110:18180-18184.

Harpole, W.S., and D. Tilman. 2006. Non-neutral patterns of species abundance in grassland communities. Ecology Letters 9:15-23.

Stenseth, N.C., W. Falck, O.N. Bjørnstad, and C.J. Krebs. 1997. Population regulation in snowshoe hare and canadian lynx: asymmetric food web configurations between hare and lynx. Proceedings of the National Academy of Sciences USA 94:5147-5152.

Strauss, S.Y. 1991. Indirect effects in community ecology: Their definition, study and importance. Trends in Ecology \& Evolution 6:206-210.

Sykes, L., L. Santini, A. Etard, and T. Newbold. 2019. Effects of rarity form on species' responses to land use. Conservation Biology 34:688-696.

Travis, J.M.J., and C. Dytham. 1999. Habitat persistence, habitat availability and the evolution of dispersal. Proceedings of the Royal Society of London B 266:723-728.

Virgós, E., R. Kowalczyk, A. Trua, A. de Marinis, J.G. Mangas, J.M. Barea-Azcón, and E. Geffen. 2011. Body size clines in the European badger and the abundant center hypothesis. Journal of Biogeography 38:1546-1556.

Waldock, C., R.D. Stuart-Smith, G.J. Edgar, T.J. Bird, and A.E. Bates. 2019. The shape of abundance distributions across temperature gradients in reef fishes. Ecology Letters 22: 685-696.

Wang, Y., M.L. Allen, and C.C. Wilmers. 2015. Mesopredator spatial and temporal responses to large predators and human development in the Santa Cruz Mountains of California. Biological Conservation 190:23-33.

Weber, M.M., R.D. Stevens, J.A.F. Diniz-Filho, and C.E.V. Grelle. 2017. Is there a correlation between abundance and environmental suitability derived from ecological niche modelling? A meta-analysis. Ecography 40:817-828. 